\title{
Elementary Methods for Calculating First and Second Moments of Simple Configurations.
}

\author{
By R. F. Muirhead, M.A., D.Se.
}

1. The term Second Moment, which is already in frequent use, as applied to lines, areas and volumes, as well as masses, is preferable to the older term Moment of Inertia which properly applies only to masses.

2. The First Moment of a body, or of a volume, or of an area, or in fact of anything which can be conceived as composed of elements having magnitude and position, is defined with reference to a plane, an axis, or a point by the symbol $\Sigma(e . r)$ where $e$ is the magnitude of an element, and $r$ the measure of its distance from the plane, axis, or point of reference. The first of these quantities, the planar First Moment, is the most important, though in the case of plane figures the moment as to a line in the plane is identical with the moment as to a perpendicular plane passing through the line.

3. The Second Moment of a configuration is defined by the formula $\Sigma\left(e . r^{2}\right)$, and here again it may be planar, axial, or polar, according as $r$ is the distance of e from a plane, an axis, or a point. In Dynamics the Axial Second Moment is that which has most direct importance, but it is simpler to begin with the calculation of planar moments, and every Axial Second Moment is the sum of the planar Second Moments with reference to two mutually perpendicular planes having the axis as their line of intersection.

\section{First Moments and Centres of Inertia.}

4. The Centre of Inertia or Centroid of a system of elements $\Sigma e$ is defined as that point whose distance $\vec{x}$ from any plane of reference is given by $\bar{x} . \Sigma e=\Sigma(e \cdot x)$.

The unique existence of such a point admits of easy proof. It is obvious, too, that for similar configurations similarly situated with reference to the plane of reference, $\bar{x}$ will be proportional to their linear dimensions. 
5. Consider now a uniform straight line or thin $\operatorname{rod} A B$ whose mid point is $\mathrm{C}$, whose length is $a$ and whose mass is $m$. Taking moments with reference to the plune through $A$ perpendicular to $A B$, and equating the moment of $\mathrm{AB}$ to the sum of the moments of $\mathrm{AC}$ and $\mathrm{CB}$, we get, if $\bar{x}$ be the distance of the centroid of $\mathrm{AB}$ from $\mathrm{A}$,

$$
\begin{gathered}
m \tilde{x}=\frac{m}{2} \cdot \frac{\bar{x}}{2}+\frac{m}{2}\left(\frac{\bar{x}}{2}+\frac{a}{2}\right) \\
\therefore \quad \bar{x}=\frac{a}{2} .
\end{gathered}
$$

Thus the centroid of a uniform straight line is its mid-point.

6. If we divide a rectangle into four similar rectangles each containing $\frac{1}{4}$ of its area, or a cuboid into eight similar cuboids each containing $\frac{1}{8}$ of its volume, and equate the moment of the whole to the sum of the moments of the parts with reference to a side of the rectangle or a face of the cuboid, we shall find the mid-point of the figure to be its centroid. And, more generally, if $h$ be the height of any right or oblique prismatic body and $\bar{x}$ the distance of its centroid from one end, we shall find $\bar{x}=\frac{h}{2}$ by considering the prism as made up of an infinite number of thin uniform rods each of which has its centroid at distance $\frac{h}{2}$ from the end.

7. Next take a uniform triangular lamina $A B C$ of mass $m$, such that the perpendicular from $\mathrm{A}$ on $\mathrm{BC}$ is of length $h$, and consider its first moment as to the plane through $A$ perpendicular to the altitude $h$. Let D, E, F be the mid-points of the sides BC, CA, AB respectively, and let $\bar{x}$ be the distance of the centroid from the plane of reference. Then the four triangles AFE, FBD, EDC, DEF have their centroids respectively at distances

$$
\frac{\bar{x}}{2}, \frac{h}{2}+\frac{\bar{x}}{2}, \frac{h}{2}+\frac{\bar{x}}{2}, \dot{h}-\frac{\bar{x}}{2} .
$$

Using the same principle as before, we get

$$
\begin{gathered}
m \bar{x}=\frac{m}{4}\left\{\frac{\bar{x}}{2}+2\left(\frac{h}{2}+\frac{\bar{x}}{2}\right)+h-\frac{\bar{x}}{2}\right\} \\
\therefore \quad x=\frac{2 h}{3} .
\end{gathered}
$$


8. Consider next a uniform tetrahedron $\mathrm{OABC}$, such that $\mathrm{OA}, \mathrm{OB}, \mathrm{OC}$ are mutually perpendicular, the length of $\mathrm{OA}$ being $h$.

Let $\mathrm{D}, \mathrm{E}, \mathrm{F}, \mathrm{H}, \mathrm{K}, \mathrm{L}$ be the mid-points of $\mathrm{BC}, \mathrm{CA}, \mathrm{AB}, \mathrm{OA}$, $O B, O C$ respectively, and $P$ the 8 th corner of the cuboid whose other 7 corners are $\mathrm{O}, \mathrm{H}, \mathrm{K}, \mathrm{L}, \mathrm{D}, \mathrm{E}, \mathrm{F}$. Then the tetrahedron $O A B C$ together with the snaller one PDEF make up the three tetrahedra AFHE, BFKD, CDLE and the cuboid mentioned. Hence, taking moments as to the plane through $\mathrm{A}$ parallel to $\mathrm{OBC}$, we have

$$
\begin{gathered}
m \bar{x}+\frac{m}{8}\left(h-\frac{\bar{x}}{2}\right)=\frac{m}{8}\left\{\frac{\bar{x}}{2}+2\left(\frac{h}{2}+\frac{\bar{x}}{2}\right)\right\}+\frac{6 m}{8} \cdot \frac{3 h}{4} \\
\therefore \quad \bar{x}=\frac{3 h}{4} .
\end{gathered}
$$

Second Monents and Radii of Gyration.

9. We shall apply to the same set of figures as above the same principle that the sum of the moments of the parts is equal to the moment of the whole; but in place of the theorem of the unique centroid we shall use the Theorem of Parallel Planes which is analogous to Huyghens' Theorem of Parallel Axes, viz.,

$$
\Sigma_{m} x^{2}=\bar{x}^{\prime 2} \Sigma_{m}+\Sigma_{m x^{\prime 2}}, \text { where } x=\bar{x}+x^{\prime} \text {. }
$$

And in place of the theorem that for similar figures similarly placed, $\bar{x}$ is proportional to the linear dimensions, we have to note that for equi-dense similar figures similarly situated as to the plane of reference, $\sum_{e x}$ is proportional to the 3rd, 4 th or 5th power of a length according as the contguration is one-dimensional, twodimensional, or three-dimensional.

10. Denoting by $M$ the Second Moment of the unform rod previously described, as to a plane through the centroid parallel to the original plane of reference, we have

$$
\begin{gathered}
\mathrm{M}+m\left(\frac{a}{2}\right)^{2}=\frac{m}{2}\left\{\left(\frac{a}{4}\right)^{2}+\left(\frac{3 a}{4}\right)^{2}\right\}+2 \frac{\mathrm{M}}{8} \\
\therefore \mathrm{M}=\frac{m a^{2}}{12} .
\end{gathered}
$$

It is obvious that the same result would apply to any prismatic body referred to the plane at one end; and this, too, whether the prism is right or oblique, if we take $a$ to denote the perpendicular height of the prism. 
11. Similarly, for the triangle, taking moments as to the same plane of reference, and denoting by $M$ its Second Moment as to a parallel plane through the centroid, we have

$$
\begin{gathered}
\mathrm{M}+m\left(\frac{2 h}{3}\right)^{2}=\frac{\mathrm{M}}{16} \times t+\frac{m}{4}\left\{\left(\frac{h}{3}\right)^{2}+2\left(\frac{5 h}{6}\right)^{2}+\left(\frac{2 h}{3}\right)^{2}\right\} \\
\therefore \quad \mathrm{M}=\frac{m h^{2}}{18} .
\end{gathered}
$$

Hence the Second Moment as to the parallel plane through $\mathbf{A}$ is

$$
\frac{m h^{2}}{18}+m\left(\frac{2 h}{3}\right)^{2}=m \frac{h^{2}}{2} \text {. }
$$

12. Again the corresponding equation for the tetrahedron gives

$$
\begin{gathered}
\mathrm{M}+m\left(\frac{3 h}{4}\right)^{2}+\frac{\mathrm{M}}{32}+\frac{m}{8}\left(\frac{5 h}{8}\right)^{2} \\
=3 \frac{\mathrm{M}}{32}+\frac{m}{8}\left(\frac{3 h}{8}\right)^{2}+2 \frac{m}{8}\left(\frac{7 h}{8}\right)^{2}+\frac{3 m}{4} \frac{h^{2}}{48}+\frac{3 m}{4}\left(\frac{3 h}{4}\right)^{2} \\
\therefore \quad \mathrm{M}=\frac{3 m h^{2}}{80} .
\end{gathered}
$$

Hence the Second Moment as to the plane of reference through A is equal to $\frac{3 m h^{2}}{80}+m\left(\frac{3 h}{4}\right)^{2}=\frac{3 m h^{2}}{5}$; and as to the plane of the base it is $\frac{3 m h^{2}}{80}+m\left(\frac{h}{4}\right)^{2}=\frac{m h^{2}}{10}$.

It is obvious that these formulie will apply to any pyramid whatever, taken with reference to planes parallel to the base; for such a pyramid could be dissected into infinitesimal triangular pyramids with the same apex and height, and it is obvious that, with reference to such a plane, an oblique tetrahedron has the same Second Moment as a right one with equal base and height.

13. The Second Moment of a uniform circular lamina of mass $m$ and radius $r$, about a perpendicular axis through its centre can now be found by supposing it dissected into infinitesimal triangles of mass $e$, whose vertices are at the centre. For each of these triangles the Second Moment relative to this axis is the same as that relative to a plane through this axis perpendicular to the mid line of the triangle, and is therefore $e r^{2} / 2$. 
Hence the required Second Moment of the lamina is $\searrow\left(e r^{2} / 2\right)$ and therefore $=m r^{2} / 2$.

Using the theorem (1) we at once find the Second Moment of the disc relative to any plane through its axis to be $m r^{2} / t$, and this is of course the same as its Second Moment as to a diameter of the circle as axis.

14. It is clear that for a uniform solid cylinder, which may be looked on as composed of uniform thin circular discs, the Second Moment with reference to its axis is also $m r^{2} / 2$. As it is prismatic, its Second Moment as to the plane of one end is $\frac{1}{3} m h^{2}$; while as to a diameter of the end, its axial Second Moment, by (1), is $\frac{m h^{2}}{3}+\frac{m r^{2}}{4}$.

15. Again, if a uniform solid sphere be dissected into infinitesimal tetrahedra each having mass $e$ and a vertex at the centre, the planar Second Moment of any tetrahedron as to a diametral plane perpendicular to its length, and therefore its polar Second Moment as to the centre, would be $3 e r^{2} / 5$.

Hence the polar Second Moment of the solid sphere as to the centre is $Y\left(e r^{2} / 5\right)$ or $3 m^{2} / 5$; from which it follows that the Second Moment as to a diametral plane is $m r^{2} / 5$, and that as to a diameter is $2 m r^{2} / 5$.

16. By the aid of the well-known and obvious principle that a pure longitudinal strain applied to a configuration in a direction perpendicular to the plane of reference, without change of mass, will increase the Second Moment as to that plane in proportion to the square of a length in that direction, we can at once extend the results for circles and cylinders and spheres so as to apply to ellipses and elliptic cylinders and ellipsoids, so far as their principal planes and axes are concerned.

17. It is perhaps worth noting that if a body be symmetrical as to a plane, or as to some axis or point in that plane, the Second Moment of one of the two halves into which it is divided by the plane in question, taken with reference to that plane, is exactly half of the Second Moment of the whole body. In this way we can, for example, get the second Moments of a semicircular lamina as to its 
diameter, of the quadrant of a circle with reference to a radial boundary as axis, and those of the hemisphere, of the quadrant of a solid sphere, and of the octant of a solid sphere, with reference to their plane faces.

18. To find the Second Moment of a uniform solid right circular cone about its axis, consider a right circular cylinder whose length is equal to its diameter. The sphere inscribed in this cylinder is equal to the "remainder" of the cylinder when a double cone has been taken away whose bases are the ends of the cylinder. For if we take any coaxial cylindric surface and the inscribed sphere, the surface of the latter is obviously equal to that part of the cylindric surface which lies within the "remainder." Hence if the "remainder" and the sphere be divided into elementary shells by an infinite number of such surfaces, the corresponding elements will be equal, and each element of the "renuinder" will have the same Second Moment as to the axis of the cylinder as the corresponding splerical element has as to the centre.

Thus the axial Second Moment of the "remainder" about its axis is equal to the polar second Moment of the sphere as to its centre, viz., $4 m \times 3 r^{2} / 5$, where $m$ is the mass of one half of the double cone. (This result might also have been arrived at without reference to the sphere, by dividing the "remainder" into infinitesimal pyramids with their vertices at the apex of the double cone).

Now the Second Moment of the whole cylinder (of mass $6 \mathrm{~m}$ ) about its axis is $6 m \times r^{2} / 2$. This leaves $3 m^{3}-12 m r^{2} / 5=3 m r^{2} / 5$ as the moment of the double cone about its axis.

Hence the Second Moment about its axis of the single cone is $\frac{: 3}{10} m r^{2}$, and this will, by Art. 16, clearly hold good whether the leeight is equal to the radius of the base, or not. The Second Moment as to an axial plane will be $\frac{3}{20} m r^{2}$, and this can be extended by the principle of Art. 16 to the Second Moment of a right elliptic cone taken as to a principal axial plane, if $r$ stands for the semiaxis of the base which is perpendicular to the plane of reference.

Again, since a right circular cone is a species of pyramid, we see that its Second Moment as to a plane through its apex parallel to its base is $3 m h^{2} / 5$, and as to the plane of its base $m h^{2} / 10$, so that the axial Second Moment as to a diameter of the base is

$$
\left(3 m r^{2}+2 m h^{2}\right) / 20 \text {. }
$$


19. The idea of using Huygens' Theorem of Parallel Axes or its analogue for planar moments to find Second Moments without integration is not new-in fact, its application to the case of the rod has long been common property, and I find that there is a paper by Rehfeld in Grunert's Archiv, 2nd Series, Vol. 16, in which the same idea is developed and applied to most of the cases treated in the present article. So far as I am aware, however, no account of the method has hitherto been published in English. Considering this, and the fact that my treatment of the topic differs a good deal from that of Rehfeld's paper, I have ventured to offer the present communication to the Society. 